\title{
Fatores preditivos de resposta ao tratamento com inibidores de protease em um centro na Amazônia ocidental
}

\section{Predictor factors of response to treatment with protease inhibitors in a center of the western Amazon}

Miguel Yasuo Tomita Nicacio', Alberto Pereira Firmino Filho', Everton Felipe do Vale Araújo', Melquior Brunno Mateus de Matos ${ }^{1}$, Thayrine Medeiros Milhomem², Cirley Maria de Oliveira Lobato ${ }^{3}$.

\section{RESUMO}

Introdução: Cerca de 170 milhões de pessoas no mundo vivem com o vírus da hepatite C. Os inibidores de proteases (Boceprevir e Telaprevir) foram os primeiros antivirais de ação direta capazes de apresentar taxas de Resposta Virológica Sustentada (RVS) mais elevadas do que aquela alcançada pela terapia dupla. Entretanto, estiveram disponíveis por curto período dificultando estudos clínicos prolongados. Objetivo: Avaliar a eficácia terapêutica da terapia tripla no tratamento da hepatite $C$ crônica genótipo 1. Material e métodos: Estudo transversal que utilizou registros médicos de pacientes com hepatite $C$ crônica tratados com inibidores de protease no Hospital das Clínicas do Acre. A análise estatística incluiu descrição de frequência (\%), médias, desvio padrão e qui-quadrado. Os valores foram estatisticamente significantes quando a probabilidade do erro alfa $(\alpha)$ era menor que $5 \%(p<0.05)$. Resultados: Dos 102 pacientes que usaram inibidores de proteases, 68 completaram o tratamento e 51 (75\%) alcançaram RVS, estando significativamente associada aos valores de carga viral e de exames laboratoriais realizados durante o tratamento como hemoglobina, hematócrito, aspartato aminotransferase, alanina aminotransferase e gama glutamil transferase. Fosfatase alcalina prétratamento também apresentou correlação relevante. Os valores de PCR-RNA abaixo do ponto de corte foram relevantes desde o período pré-tratamento até a 48ำ semana. Conclusão: A terapia com inibidores de protease foi eficaz e segura na maioria dos pacientes. Contudo, efeitos colaterais e baixa taxa de RVS permanecem como fatores limitantes, minimizados com a introdução dos novos antivirais de ação direta.

Descritores: Hepatite C. Resposta Virológica Sustentada. Eficácia.

\section{ABSTRACT}

Introduction: About 170 million people around the globe live with the Hepatitis C Virus. Proteases Inhibitors (Boceprevir and Telaprevir) were the firsts direct acting antivirals developed whose efficiency was ensured by raising the rates of Sustained Virological Response (SVR) when compared to the dual therapy. But they were available for a short time hampering long term studies. Objective: evaluate the therapeutic effectiveness of triple therapy with Pegylated Interferon and Ribavirin combined with Boceprevir or Telaprevir in the treatment of chronic hepatitis C in genotype 1 patients. Methods: Cross-sectional study using medical records of patients with chronic hepatitis $\mathrm{C}$ who underwent therapy with PI treated at Hospital das Clínicas do Acre. The analyses description included frequencies (\%), means, standard deviation and chisquare test. The values were significantly relevant when the probability of the alpha ( $\alpha)$ error was less than $5 \%(p<0.05)$. Results: Of the 102 patients who used PI, 68 completed treatment and $51(75 \%)$ achieved SVR. This SVR was significantly associated to the viral load and some laboratory tests such as hemoglobin and hematocrit values, aspartate aminotransferase, alanine aminotransferase, and gamma glutamyl transferase during treatment. The alkaline phosphatase pretreatment value was relevant as well. The PCR-RNA results below the cut-off point were relevant since the pre-treatment period until the 48th week. Conclusion: Proteases Inhibitors antiviral therapy was effective and safe in most patients. However, side effects and low SVR rate remain as limiting factors, which are being minimized with the introduction of new direct acting antiviral agents.

Descriptors: Hepatitis C. Sustained Virological Response. Efficacy.
${ }^{1}$ Acadêmico de medicina da

Universidade Federal do Acre,

Rio Branco, Acre, Brasil.

${ }^{2}$ Médica

formada

pela

Universidade Federal do Acre.

Residente em pediatria do

Hospital das Clínicas do Acre.

Rio Branco, Acre, Brasil.

${ }^{3}$ Médica formada pela

Universidade do Amazonas-

Faculdade de Ciências da

Saúde. Professora Adjunta do Centro da Saúde e do Desporto da Universidade Federal do Acre. Doutorado em Saúde Pública pela Universidade de São Paulo, USP, Brasil.

Estudo realizado com apoio financeiro da Universidade Federal do Acre

Endereço para

correspondência:

Miguel Yasuo Tomita Nicacio. Rua Vitória 601, Floresta, 69915-232 Rio Branco, Acre, Brasil. Phone: 5568 32272439; Mobile: 5568 999759866. 


\section{INTRODUÇAOO}

Dados da Organização Mundial da Saúde (OMS) ${ }^{1}$ apontam, mundialmente, cerca de 170 milhões de portadores do vírus da hepatite C - VHC. No Brasil, aproximadamente 10.000 casos são notificados a cada ano ${ }^{2}$. O VHC é um vírus RNA com alta taxa de replicação, e variável grau de dano hepático ${ }^{3,4}$. Dentre os objetivos do tratamento, temos a interrupção da agressão hepática e alcançar a resposta virológica sustentada (definida como PCR-RNA indetectável após 6 meses do término do tratamento) ${ }^{4}$, estando estes objetivos relacionados a fatores virais e as características clínicas do paciente ${ }^{5,6}$.

Até o ano de 2012, o esquema recomendado para tratamento dos pacientes era a associação de Interferon (IFN) e Ribavirina (RBV) ${ }^{7}$. Entretanto, foi observado uma alta prevalência de efeitos colaterais e baixa resposta ao tratamento, principalmente, nos pacientes com genótipo $1^{8}$. Em 2011, a American Association for the Study of Liver Diseases tornou público um guia com indicações para o uso de Inibidores de Protease (IP) em pacientes portadores de HCV genótipo 1, fato que contribuiu para uma melhora significativa nas taxas de Resposta Virológica Sustentada (RVS) ${ }^{9}$.

Os primeiros inibidores de proteases desenvolvidos foram o Boceprevir (BOC) e o Telaprevir (TVR) ${ }^{2}$. Ambos utilizados em associação com Interferon Peguilado e Ribavirina, constituindo, assim, uma terapia tripla ${ }^{10,11}$. Desde então, a eficácia dos IP sobre a replicação e montagem do HCV genótipo 1 foi reafirmada ao elevar o número de pacientes com RVS ${ }^{10,12}$. Em 2015, a atualização do protocolo de tratamento do Ministério da Saúde descontinuou o uso dos IP de primeira geração. Por outro lado, foram adicionados ao arsenal terapêutico do SUS os Antivirais de Ação Direta (DAAS), que atuam diretamente no HCV, interrompendo a sua replicação e constituem avanço recente no tratamento da hepatite C crônica ${ }^{2,13-15}$.

Por tudo isso, constata-se que a vigência terapêutica dos IP de primeira geração foi rápida, dificultando o estudo clínico em longo prazo. Assim, a presente pesquisa tem como objetivo avaliar a eficácia terapêutica da terapia tripla no tratamento da Hepatite Crônica pelo Vírus C nos pacientes genótipo 1 atendidos no Serviço de Assistência Especializado. Bem como, verificar as características desses pacientes, os fatores associados à resposta virológica sustentada e o impacto dessas drogas na terapêutica da hepatite C no Estado. 
Estudo transversal, em que foram analisados os prontuários dos pacientes portadores crônicos de HCV genótipo 1, submetidos à terapia com IP, atendidos no Serviço de Assistência Especializada do Hospital das Clínicas do Acre, localizado na cidade de Rio Branco durante o período de 2012 a 2015. Foram excluídos os pacientes que não concluíram a terapia tripla até a $48^{\mathrm{a}}$ semana.

Do total de 102 pacientes submetidos ao tratamento com IP, 68 concluíram o tratamento. Os pacientes foram avaliados quanto às características demográficas e epidemiológicas (idade, sexo, estado civil, procedência, entre outras). Quanto aos parâmetros laboratoriais e a carga viral foram coletados os exames nos períodos prétratamento, na $4^{\circ}, 12^{\circ}, 48^{\circ}$ semana de tratamento.

Os exames de imagem foram coletados no período pré-tratamento. As características clínicas foram avaliadas antes do início do e durante o tratamento, relacionadas ao genótipo viral, história de alcoolismo e tabagismo, e durante o tratamento foram avaliados os efeitos colaterais apresentados.

Nessa análise, foram estabelecidas as frequências (\%), médias e desvio padrão para as variáveis demográficas, epidemiológicas, clínicas e exames de imagem. O teste qui-quadrado foi utilizado para avaliar o grau de associação entre a RVS e as características clínicas do paciente, exames laboratoriais e a carga viral.

Para o teste qui-quadrado, os resultados dos exames laboratoriais e a carga viral foram recategorizados em duas variáveis, de acordo com o ponto de referência estabelecido pelo laboratório de exames laboratoriais do Hospital das Clínicas do Acre, levando-se a diferenças entre os sexos. Os valores foram considerados como significativamente relevantes quando o valor da probabilidade do erro alfa $(\alpha)$ foi inferior a $5 \%(p<0,05)$.

Este estudo foi conduzido em concordância com todos os aspectos éticos, em relação às recomendações estabelecidas pelo Comitê de Ética em Pesquisa, conforme expresso na Resolução CNS no 486/2012. O projeto original deste estudo foi aprovado pelo Comitê de Ética em Pesquisa do Hospital das Clínicas Acre. Registro CAAE: 11164612.0.3001.5009. 
Com relação aos 68 pacientes que concluíram o tratamento, evidencia-se predomínio do sexo masculino 56 (76,7\%), com média de idade de 55 anos (DP $\pm 7,6)$, procedentes em sua maioria da capital Rio Branco 48 (70,6\%). Com relação a história clínica, 31 (45,6\%) eram cirróticos, 26 (38,2\%) tinham esteatose hepática, e 4 (5,9\%) eram transplantados de fígado.

O subgenótipo viral mais prevalente foi o $1 \mathrm{~A}$ com 37 (54,4\%) pacientes, seguido pelo 1B com 18 (26,5\%), 13 (19,1\%) pacientes não tiveram o subgenótipo especificado. O tempo médio de acompanhamento dos pacientes até o início do tratamento foi de 74 meses (DP $\pm 60,245)$. Com relação ao tratamento, $37(54,4 \%)$ já haviam sido tratados previamente com terapia dupla. Já na terapia tripla, $61(89,7 \%)$ utilizaram telaprevir e 7 $(10,3 \%)$ boceprevir.

Dos 68 pacientes que concluíram as 48 semanas de terapia tripla, $75 \%(n=51)$ alcançaram Resposta Virológica Sustentada (RVS), ou seja, mantiveram carga viral indetectável 24 semanas após o final do tratamento. A RVS foi maior nos pacientes que utilizaram telaprevir com $77 \%(n=74)$ e nos que utilizaram boceprevir foi de $57,1 \%(n=4)$. Contudo, não foi encontrado um grau de associação significamente estatístico $(p<0,05)$ entre as variáveis relacionadas ao paciente e uma melhor RVS, como mostrado na Tabela 01.

Comparando-se os exames laboratoriais e a carga viral, com a presença de RVS, Tabela 02, mostraram associação relevante $(p<0,05)$ valores de hemoglobina $\mathrm{e}$ hematócrito na $12^{\circ}$ semana, valores de TGO a partir da $4^{\circ}$ semana, TGP a partir da $12^{\circ}$ semana. A presença de Fosfatase Alcalina sem alterações previamente à terapêutica, na $4^{\circ}$ e $12^{\underline{a}}$ semana de tratamento também se mostrou relevante quanto à obtenção de RVS. Os valores de gama GT na $12^{\circ}$ e $48^{\circ}$ semana também se mostraram relevantes, assim como os valores de bilirrubina total na $48^{\circ}$ semana.

Os resultados de PCR abaixo do ponto de corte, mostraram-se relevantes desde o período pré-tratamento até a $48^{\circ}$ semana. As demais associações não apresentaram significância favorável.

Com relação aos exames de imagem, 45 haviam realizado Ultrassonografia no período pré-tratamento, e os principais achados nos laudos foram: fígado heterogêneo em 19 (42,2\%), hepatomegalia em 13 (28,9\%), nódulos hepáticos em 9 (20\%) e esplenomegalia em 8 (17,8\%) pacientes. Já na Endoscopia Digestiva Alta (EDA), 36 haviam realizado o exame, as varizes de esôfago estavam presentes em 36 (88,2\%) 
principalmente, de fino calibre, além disso 20 (55,6\%) tinham gastrite, 7 (10,3\%) eram positivos para Helicobater pylori e $3(8,3 \%)$ apresentavam sinais de hipertensão gástrica.

Durante o tratamento 49 (72\%) pacientes apresentaram algum efeito colateral ao tratamento, dos quais destacam-se: astenia, dor abdominal, prurido, mialgia, cansaço em membros inferiores e náuseas.

Tabela 1: Relação entre as características clínicas de Resposta Virológica Sustentada

\begin{tabular}{|c|c|c|c|c|}
\hline Característica laboratorial & Sem resposta $(\%)$ & Com resposta $(\%)$ & $p$ & Perdidos \\
\hline \multicolumn{5}{|l|}{ Hemoglobina } \\
\hline $12^{\circ}$ semana & $15(24,2)$ & $47(75,8)$ & 0,038 & 6 \\
\hline \multicolumn{5}{|l|}{ Hematcrito } \\
\hline $12^{\circ}$ semana & $15(24,2)$ & $47(75,8)$ & 0,026 & 6 \\
\hline \multicolumn{5}{|l|}{$\overline{\text { AST }}$} \\
\hline $4^{\circ}$ semana & $15(24,2)$ & $47(75,8)$ & 0,017 & 6 \\
\hline $12^{\circ}$ semana & $14(23,3)$ & $46(76,7)$ & 0,006 & 8 \\
\hline \multicolumn{5}{|l|}{ ALT } \\
\hline $12^{\circ}$ semana & $15(24,6)$ & $47(75,4)$ & 0,033 & 7 \\
\hline \multicolumn{5}{|l|}{ Fosfatase alcalina } \\
\hline Pre-tratamento & $15(24,6)$ & $46(75,4)$ & 0,019 & 7 \\
\hline $4^{\circ}$ semana & $14(25,92)$ & $40(74,08)$ & 0,011 & 14 \\
\hline $12^{\circ}$ semana & $12(22,33)$ & $42(77,77)$ & 0,009 & 14 \\
\hline \multicolumn{5}{|l|}{ Gama-GT } \\
\hline $12^{\circ}$ semana & $14(24,1)$ & $44(75,9)$ & 0,026 & 10 \\
\hline \multicolumn{5}{|l|}{ VHC-RNA } \\
\hline Pre-tratamento & $15(25)$ & $45(75)$ & 0,023 & 8 \\
\hline $4^{\circ}$ semana & $16(25,4)$ & $47(74,6)$ & 0,001 & 5 \\
\hline $12^{\circ}$ semana & $16(26,3)$ & $45(73,77)$ & 0,002 & 7 \\
\hline
\end{tabular}

Tabela 2: Relação entre as características laboratoriais de Resposta Virológica Sustentada

\begin{tabular}{|c|c|c|c|c|}
\hline Característica laboratorial & Sem resposta $(\%)$ & Com resposta(\%) & $p$ & Perdidos \\
\hline \multicolumn{5}{|l|}{ Hemoglobina } \\
\hline $12^{\circ}$ semana & $15(24,2)$ & $47(75,8)$ & 0,038 & 6 \\
\hline \multicolumn{5}{|l|}{ Hematcrito } \\
\hline $12^{\circ}$ semana & $15(24,2)$ & $47(75,8)$ & 0,026 & 6 \\
\hline \multicolumn{5}{|l|}{ AST } \\
\hline $4^{\circ}$ semana & $15(24,2)$ & $47(75,8)$ & 0,017 & 6 \\
\hline
\end{tabular}




\begin{tabular}{|c|c|c|c|c|}
\hline $12^{\circ}$ semana & $14(23,3)$ & $46(76,7)$ & 0,006 & 8 \\
\hline \multicolumn{5}{|l|}{ ALT } \\
\hline $12^{\circ}$ semana & $15(24,6)$ & $47(75,4)$ & 0,033 & 7 \\
\hline \multicolumn{5}{|l|}{ Fosfatase alcalina } \\
\hline Pre-tratamento & $15(24,6)$ & $46(75,4)$ & 0,019 & 7 \\
\hline $4^{\circ}$ semana & $14(25,92)$ & $40(74,08)$ & 0,011 & 14 \\
\hline $12^{\circ}$ semana & $12(22,33)$ & $42(77,77)$ & 0,009 & 14 \\
\hline \multicolumn{5}{|l|}{ Gama-GT } \\
\hline $12^{\circ}$ semana & $14(24,1)$ & $44(75,9)$ & 0,026 & 10 \\
\hline \multicolumn{5}{|l|}{ VHC-RNA } \\
\hline Pre-tratamento & $15(25)$ & $45(75)$ & 0,023 & 8 \\
\hline $4^{\circ}$ semana & $16(25,4)$ & $47(74,6)$ & 0,001 & 5 \\
\hline $12^{\circ}$ semana & $16(26,3)$ & $45(73,77)$ & 0,002 & 7 \\
\hline
\end{tabular}

\section{DISCUSSÃO}

De acordo com o boletim epidemiológico de hepatites virais, o Brasil vem apresentando um crescimento na taxa de detecção de casos de hepatite $\mathrm{C}$ com anti-HCV e HCV-RNA positivos. Ainda de acordo com este boletim, desde 1999, 152.712 casos de hepatite $C$ foram notificados, tendo uma pequena predominância pelo sexo masculino, na proporção de M:F de 1:1,3, que vem igualando-se desde 1999. Ademais, a faixa etária na qual concentra-se as mais altas prevalências é entre 50 e 59 anos. Semelhantemente, neste estudo sexo masculino demostrou-se predominante, além disso, a média de idade, permaneceu dentro da faixa etária dos 50 a 59 anos ${ }^{11}$.

Levando-se em consideração a RVS, neste estudo foi possível verificar uma taxa de RVS em $75 \%$ dos pacientes, fato que está de acordo com o que é encontrado na litura, no qual a RVS varia entre taxas de $60 \%$ a $80 \%^{16-20}$, variando de acordo com as características prévias do paciente, das quais a presença de cirrose hepática tem-se mostrado como um dos principais fatores, apesar de não ter tido relevância estatística neste estudo.

Um estudo SPRINT-2 ${ }^{17}$, com 1.097 pacientes virgens de tratamento para VHC genótipo 1, que utilizaram o esquema de tratamento composto por Interferon, Ribavirina e boceprevir durante 48 semanas, tiveram uma taxa de RVS de $66 \%$, enquanto um estudo ADVANCE ${ }^{16}$, com 1.095 virgens de tratamento para VHC genótipo 1, demostrou uma taxa de RVS $75 \%$ dos pacientes tratados com terapia tripla, utilizando telaprevir no esquema 
durante as 48 semanas. Resultados semelhantes foram encontrados nos pacientes previamente tratados ${ }^{18,19}$. Do mesmo modo, neste estudo foi possível identificar uma maior RVS nos pacientes que utilizaram telaprevir, ademais verifica-se que a maioria dos esquemas de tratamento utilizaram telaprevir em detrimento de boceprevir, prejudicando o estabelecimento de uma relação estatíscamente significativamente entre o inibidor de protease utilizado e uma melhor taxa de RVS.

Uma melhor taxa de RVS é influenciada por diversos fatores, que podem ser intrínsecos ou extrínsecos ao paciente. Um estudo conduzido com pacientes que utilizaram terapia tripla, alguns fatores foram associados a melhores taxas de RVS, dos quais valores de VHC-RNA $<800.000 \mathrm{UI} / \mathrm{mL}$ pré-tratamento estiveram relacionados a uma melhor taxa de RVS 20,21 . Neste estudo, a análise dos valores de VHC-RNA prétratamento e durante as primeiras semanas de tratamento, foi possível identificar uma relação estatisticamente significante entre valores mais baixos de VHC-RNA prétratamento, Resposta Virológica Rápida e Precoce com melhores taxas de RVS, justificando assim, a necessidade de acompanhamento da carga viral dos pacientes, a fim de verificar parâmetros para o estabelecimento de falha terapêutica, bem como do conhecimento de fatores preditivos positivos para RVS.

Durante a avaliação para a indicação para o tratamento é importante verificar a presença de preditores de melhor resposta ao tratamento. Com relação aos preditores pré-tratamento, além da carga viral, alguns estudos vêm buscando estabelecer relação com exames laboratoriais, a saber, AFP, plaquetas (>150.000), hemoglobina e provas de função e lesão hepática. Um estudo conduzido com pacientes em uso de telaprevir, estabeleceu relações significativamente relevante, entre RVS e valores de AFP $<10 \mu \mathrm{g} / \mathrm{L}$, plaquetas $>150.000^{21}$. Assim como em nossa pesquisa, um estudo com 149 pacientes tratados com inibidores de protease, não encontraram relações importantes entre os valores dos exames laboratoriais pré-tratamento e melhores taxas de RVS, exceto pelos valores de $A F P<10 \mu \mathrm{g} / \mathrm{L}^{20}$. Contudo, em nosso estudo, pacientes com valores abaixo do nível superior de normalidade de fosfatase alcalina pré-tratamento obtiveram maiores taxas de RVS, possivelmente, devido ao fato que a presença de valores mais elevados de enzimas canaliculares em portadores de VHC crônico represente estágios mais avançados da doença, dificultando uma melhor resposta ao tratamento.

O acompanhamento laboratorial durante o tratamento é importante para avaliar a normalização dos valores de prova de lesão hepática. Diversos estudos comprovam a importância da avaliação dos valores de aminotransferases pré-tratamento e durante, 
devido a relação que possui com melhores taxas de RVS. Um estudo avaliando 168 pacientes em tratamento com terapia dupla, foi demonstrado a relação entre uma melhor RVS nos pacientes que normalizaram os valores de ALT nas primeiras 4 semanas de tratamento ${ }^{22}$. Do mesmo modo, em nosso estudo, foi possível verificar melhores taxas de RVS em pacientes que normalizaram os níveis de AST, ALT, FA e Gama-GT durante as primeiras 12 semanas de tratamento, revelando a importância de uma menor atividade necro-inflamatória para uma melhor resposta ao tratamento.

Com relação aos efeitos adversos, estudos vem mostrando uma alta porcentagem de pacientes que apresentam anemia, chegando a atingir $50 \%$ dos pacientes em determinado estudos ${ }^{23}$. A anemia já vem sendo relatada ao uso de terapias compostas por INF/RBV, contudo com a incorporação do inibidor de protease este efeito adverso esteve presente em uma maior quantidade de pacientes ${ }^{11}$. No presente estudo, a anemia esteve presente em 16 pacientes durante o tratamento e 2 interromperam o tratamento por anemia severa.

Os esquemas terapêuticos compostos por INF/RBV, geralmente, apresentam efeitos colaterais nos pacientes em uso da medicação, o que muitas vezes pode levar a interrupção do tratamento. Neste estudo, $72 \%$ dos pacientes apresentaram algum sintoma durante o tratamento. Dentre os sintomas apresentados, a astenia esteve presente em cerca de $25 \%$ dos pacientes, comumente, apresentado nos pacientes em uso de INF/RBV, assim como mialgia ${ }^{24}$. O prurido é um dos sintomas cutâneos mais frequentes em pacientes em uso de INF/RBV, porém outras manifestações cutâneas, como rash, não apresentaram grande relevância neste estudo, apesar do fato de estar presente em grande parte dos pacientes em uso de telaprevir 25 .

Em um estudo conduzido com 467 pacientes, 25,9\% dos pacientes descontinuaram o tratamento, dos quais a maioria foi devido a falha terapêutica e a graves efeitos adversos ${ }^{25}$. Neste estudo, dentre os pacientes que descontinuaram o tratamento, pode-se observar um predomínio por falha terapêutica, seguido por efeitos adversos, semelhante ao que é encontrado na literatura. Sendo este, um dos principais fatores limitantes para o estabelecimento deste esquema terapêutico como primeira escolha para 0 atual tratamento de pacientes com VHC genótipo 1, que se soma a baixa taxa de RVS nos pacientes que concluíram a terapia, quando comparado aos resultados obtidos pelos Antivirais de Ação Direta (DAAS). 
A terapia antiviral com IP foi eficaz e segura na maioria desses pacientes hepatopatas crônico pelo vírus C, genótipo 1. No entanto, os efeitos colaterais e a baixa taxa de resposta virológica sustentada continuam sendo um fator limitador do referido tratamento, que estão sendo minimizado com a introdução dos novos agentes antivirais de ação direta (DAAS).

Embora as complicações e a falha terapêutica estiveram presentes em uma grande maioria dos pacientes, mais da metade dos pacientes incluídos neste estudo alcançou a RVS, o que justificou a decisão de 2013 de introduzir boceprevir e telaprevir no protocolo brasileiro. Porém com o surgimento de novas opções terapêuticas com maior percentual de RVS e menos efeitos colaterais, sua rápida retirada em 2015, após uma análise de custo-benefício realizada pelas autoridades de saúde pública do país, pareceu ser uma decisão boa e oportuna. Ademais, este estudo pode contribuir com o fornecimento dados conceituais e comparatórios que contribuam no processo de evolução da assistência terapêutica à Hepatite $\mathrm{C}$ crônica.

Os principais impasses foram a dificuldade em conduzir os efeitos colaterais, devido à falta insumos e medicamentos apropriados. Além disso, houve um número importante de pacientes que não concluíram o tratamento, principalmente, devido aos efeitos colaterais e a baixa resposta inicial ao tratamento.

\section{REFERÉNCIAS}

1. World Health Organization. Guidelines for the Screening, care and treatment of persons with hepatitis C infection. 2014. Available at: http://apps.who.int/iris/bitstream/10665/111747/1/9789241548755 eng.pdf?ua=1. Accessed May 20, 2017.

2. Ministério da Saúde (Brasil), Relatório de Recomendação da Comissão Nacional de Incorporação de Tecnologias no SUS - CONITEC. Protocolo clínico e diretrizes terapêuticas para hepatite C e coinfecções. Brasília: Ministério da Saúde; 2015. 89 p.

3. Neumann AU, Lam NP, Dahari H, Gretch DR, Wiley TE, Layden T, et al. Hepatitis C viral dynamics in vivo and the antiviral efficacy of interferon-aphatherapy. Science. 1998;282(5386):103-7.

4. Bukh J, Miller RH, Purcell RH. Genetic heterogeneity of hepatitis c virus: quasispecies and genotypes. Semin Liver Dis. 1995;15(1):41-63. 
5. Santos BF, de Santana NO, Franca AV. Prevalence, genotypes and factors associated with HCV infection among prisoners in Northeastern Brazil. World J. Gastroenterol. 2011;17(25):3027-34.

6. Farnik H, Lange CM, Sarrazin C, Kronenberger B, Zeuzem S, Herrmann E. Meta-analysis shows extended therapy improves response of patients with chronic hepatitis $\mathrm{C}$ virus genotype 1 infection. Clin Gastroenterol Hepatol. 2010;8(10):884-90.

7. Seeff LB. Natural history of chronic hepatitis C. Hepatology. 2002;36(5):35-46.

8. Ghany MG, Nelson DR, Strader DB, Thomas DL, Seeff LB. An update on treatment of genotype 1 chronic hepatitis $C$ virus infection: 2011 practice guideline by the American Association for the Study of Liver Diseases. Hepatology. 2011;54(4):1433-44.

9. Ministério da Saúde (Brasil), Relatório de Recomendação da Comissão Nacional de Incorporação de Tecnologias no SUS - CONITEC. Inibidores de Protease (Boceprevir e Telaprevir) para o tratamento da Hepatite Crônica C. Brasília: Ministério da Saúde; 2015. 102 p.

10. Ministério da Saúde (Brasil), Secretaria de Vigilância em Saúde. Departamento de DST, Aids e Hepatites Virais. Protocolo Clínico e Diretrizes Terapêuticas para Hepatite Viral C e Coinfecções, Suplemento 1. Manejo do paciente infectado cronicamente pelo genótipo 1 do HCV e fibrose avançada. Brasília: Ministério da Saúde; 2013. 56 p.

11. Jacobson IM, McHutchison JG, Dusheiko G, Di Bisceglie AM, Reddy KR, Bzowej $\mathrm{NH}$, et.al. Telaprevir for previously untreated Chronic Hepatitis C Virus. $\underline{\mathrm{N}}$ Engl $\mathrm{J}$ Med. 2011;364(25):2405-16

12. American Association for the Study of Liver Diseases. AASLD Practice Guidelines: Recommendations for testing, managing and treating Hepatitis C. Available at: http://www.hcvguidelines.org/. Accessed May 15, 2017.

13. Summers BB, Beavers JW, Klibanov OM. Sofosbuvir, a novel nucleotide analogue inhibitor used for the treatment of hepatitis C virus. J Pharm Pharmacol. 2014;66(12):1653-66.

14. Kohli A, Shaffer A, Sherman A, Kottilil S. Treatment of Hepatitis C. JAMA. $2014 ; 312(6): 631-40$.

15. Ministério da Saúde (Brasil), Secretaria de Vigilância em Saúde, Departamento de DST, Aids e Hepatites Virais. Boletim epidemiológico: hepatites virais. Brasília (DF): Ministério da Saúde; 2016. p 72.

16. Poordad F, McCone J Jr, Bacon BR, Bruno S, Manns MP, Sulkowski MS, et al. Boceprevir for untreated chronic HCV genotype 1 infection. N Engl J Med 2011; 364(13):1195-206.

17. Bacon BR, Gordon SC, Lawitz E, Marcellin P, Vierling JM, Zeuzem S, et al. Boceprevir for previously treated chronic HCV genotype 1 infection. $\mathrm{N}$ Engl J Med. 2011; 364(13):1207-17. 
18. Zeuzem S, Andreone P, Pol S, Lawitz E, Diago M, Roberts S, et al. Telaprevir for retreatment of HCV infection. N Engl J Med. 2011; 364(25):2417-28.

19. Kanda T, Nakamoto S, Sasaki R, Nakamura M, Yasui S, Haga Y, et al. Sustained virologic response at 24 weeks after the end of treatment is a better predictor for treatment outcome in real-world hcv-infected patients treated by hcv ns $3 / 4 \mathrm{a}$ protease inhibitors with peginterferon plus ribavirin. Int J Med Sci. 2016;13(4): 31015.

20. Colombo M, Strasser S, Moreno C, Abrao Ferreira P, Urbanek P, Fernández I, et al. Sustained virological response with telaprevir in 1078 patients with advanced hepatitis C: The international telaprevir access program. J Hepatol. 2014;61(5): 976-83.

21. Kim YJ, Jang BK, Kim ES, Park KS, Cho KB, Chung WJ, et al. Rapid normalization of alanine aminotransferase predicts viral response during combined peginterferon and ribavirin treatment in chronic hepatitis $C$ patients. Korean $J$ Hepatol. 2012;18(1): 41-7.

22. Gonzalez HC, Jafri SM, Gordon SC. Role of liver biopsy in the era of direct-acting antivirals. Curr Gastroenterol Rep. 2013;15(2): 307.

23. Garcia TJ, Lara PHS, Morimoto TP, Higasiaraguti M, Perejão AM, Ayub MA. Efeitos colaterais do tratamento da hepatite $C$ no polo aplicador do ABC. Rev Assoc Med Bras 2012; 58(5): 543-9.

24. Hézode C, Fontaine H, Dorival C, Larrey D, Zoulim F, Canva V, et al. Triple therapy in treatment-experienced patients with HCV-cirrhosis in a multicentre cohort of the French Early Access Programme (ANRS CO20-CUPIC) - NCT01514890. J Hepatol. 2013;59(3): 434-41.

25. Fernández DA, Cunill RM, Iglesias MJF, Collell XT, Gómez MV, Velasco NZ, et al. Predictive variables of sustained virological response after early discontinuation of triple therapy with telaprevir for genotype-1 HCV infection. Gastroenterol Hepatol. 2016;39(6): 377-84. 